\title{
Transcriptomic evidence for involvement of reactive oxygen species in Rhizoctonia solani AG1 IA sclerotia maturation
}

\author{
Bo Liu ${ }^{1,2}$, Haode Wang ${ }^{1}$, Zhoujie Ma ${ }^{1}$, Xiaotong Gai ${ }^{1}$, Yanqiu Sun ${ }^{1}$, Shidao He ${ }^{1}$, Xian Liu ${ }^{3}$, Yanfeng \\ Wang $^{2}$, Yuanhu Xuan ${ }^{4}$, Zenggui Gao ${ }^{\text {Corresp. } 1}$ \\ 1 Institute of Plant Immunology, Shenyang Agricultural University, Shenyang, Liaoning, China \\ 2 College of Life Sciences, Yan'an University, Yan'an, Shanxi, China \\ 3 College of Life Sciences, Shenyang Agricultural University, Shenyang, Liaoning, China \\ 4 College of Plant Protection, Shenyang Agricultural University, Shenyang, Liaoning, China \\ Corresponding Author: Zenggui Gao \\ Email address: gaozenggui@syau.edu.cn
}

Rhizoctonia solani AG1 IA is a soil-borne fungal phytopathogen that can significantly harm crops resulting in economic loss. This species overwinters in grass roots and diseased plants, and produces sclerotia that infect future crops. $R$. solani AG1 IA does not produce spores; therefore, understanding the molecular mechanism of sclerotia formation is important for crop disease control. Toward this end, to identify the genes involved in this process for the development of disease control targets, in this study, the transcriptomes of this species were determined at three important developmental stages (mycelium, sclerotial initiation, and sclerotial maturation) using an RNA-sequencing approach. A total of 5016,6433 , and 5004 differentially expressed genes (DEGs) were identified in the sclerotial initiation vs. mycelial, sclerotial maturation vs. mycelial, and sclerotial maturation vs. sclerotial initiation stages, respectively. Moreover, Gene Ontology (GO) and Kyoto Encyclopedia of Genes and Genomes (KEGG) analyses showed that these DEGs were enriched in diverse categories, including oxidoreductase activity, carbohydrate metabolic process, and oxidation-reduction processes. Twelve DEGs were further verified using reverse transcription quantitative PCR (RT-qPCR). Among the genes examined, NADPH oxidase 1 (NOX1) and superoxide dismutase (SOD) were highly induced in the stages of sclerotial initiation and maturation. In addition, the highest reactive oxygen species (ROS) production levels were detected during sclerotial initiation, and enzyme activities of NOX1, SOD, and catalase (CAT) matched with the gene expression profiles. To further evaluate the role of ROS in sclerotial formation , $R$. solani AGI IA was treated with the CAT inhibitor aminotriazole and $\mathrm{H}_{2} \mathrm{O}_{2}$, resulting in the early differentiation of sclerotia. Taken together, this study provides useful information toward understanding the molecular basis of $R$. solani AGI IA sclerotial formation and maturation, and identified the important role of ROS in these processes. 


\section{Transcriptomic evidence for involvement of reactive oxygen}

2 species in Rhizoctonia solani AG1 IA sclerotia maturation

3

4 Bo Liu ${ }^{12}$, Haode Wang ${ }^{1}$, Zhoujie Ma ${ }^{1}$, Xiaotong Gai ${ }^{1}$, Yanqiu Sun ${ }^{1}$, Shidao He ${ }^{1}$, Xian Liu ${ }^{3}$, Yanfeng Wang ${ }^{2}$, Yuan

$5 \mathrm{Hu} \mathrm{Xuan}^{4}$, Zenggui Gao ${ }^{1}$

$6{ }^{1}$ Institute of Plant Immunology, Shenyang Agricultural University, Shenyang, Liaoning, China

72 College of Life Sciences, Yan'an University, Yan'an, Shanxi, China

$8{ }^{3}$ College of Life Sciences, Shenyang Agricultural University, Shenyang, Liaoning, China

$9{ }^{4}$ College of Plant Protection, Shenyang Agricultural University, Shenyang, China

10

11 Corresponding Author:

12 Zenggui Gao

13 Shenyang Agricultural University, Shenyang, Liaoning 110866, China

14

15

Email address: gaozenggui@syau.edu.cn 


\section{ABSTRACT}

Rhizoctonia solani AG1 IA is a soil-borne fungal phytopathogen that can significantly harm crops resulting in economic loss. This species overwinters in grass roots and diseased plants, and produces sclerotia that infect future crops. $R$. solani AG1 IA does not produce spores; therefore, understanding the molecular mechanism of sclerotia formation is important for crop disease control. Toward this end, to identify the genes involved in this process for the development of disease control targets, in this study, the transcriptomes of this species were determined at three important developmental stages (mycelium, sclerotial initiation, and sclerotial maturation) using an RNA-sequencing approach. A total of 5016, 6433, and 5004 differentially expressed genes (DEGs) were identified in the sclerotial initiation vs. mycelial, sclerotial maturation vs. mycelial, and sclerotial maturation vs. sclerotial initiation stages, respectively. Moreover, Gene Ontology (GO) and Kyoto Encyclopedia of Genes and Genomes (KEGG) analyses showed that these DEGs were enriched in diverse categories, including oxidoreductase activity, carbohydrate metabolic process, and oxidation-reduction processes. Twelve DEGs were further verified using reverse transcription quantitative PCR (RT-qPCR). Among the genes examined, NADPH oxidase $1(N O X 1)$ and superoxide dismutase $(S O D)$ were highly induced in the stages of sclerotial initiation and maturation. In addition, the highest reactive oxygen species (ROS) production levels were detected during sclerotial initiation, and enzyme activities of NOX1, SOD, and catalase (CAT) matched with the gene expression profiles. To further evaluate the role of ROS in sclerotial formation, $R$. solani AG1 IA was treated with the CAT inhibitor aminotriazole and $\mathrm{H}_{2} \mathrm{O}_{2}$, resulting in the early differentiation of sclerotia. Taken together, this study provides useful information toward understanding the molecular basis of $R$. solani AG1 IA sclerotial formation and maturation, and identified the important role of ROS in these processes.

\section{INTRODUCTION}

Rhizoctonia solani is a soil-borne pathogen belonging to the class Agaricomycetes, family Ceratobasidiaceae that causes various plant diseases and attacks crops (maize, potatoes, rice, and soybean). R. solani is classified into 14 anastomosis groups (AG 1-13 and AG 1 IB) (Ogoshi 1987; Priyatmojo et al. 2001). Maize sheath blight is one of the most serious and widely distributed diseases caused by $R$. solani AG1 IA, resulting in severe yield losses in maizecultivating areas worldwide. The sclerotium is a special structure of $R$. solani that overwinters in the soil or diseased plants, and can survive under adverse conditions (e.g., low temperature) for long periods (Rush \& Lee 1983; Boland et al. 2004). Although sheath blight disease-resistant varieties of maize are available, these varieties are limited; thus, treating maize with fungicides remains the main approach to control the spread of $R$. solani in crops (Zhao et al. 2006). However, the emergence of fungicide tolerance has now made it even more difficult to control this disease. Since $R$. solani AG1 IA does not produce spores, the growth and germination of sclerotial are the key to maintaining the life of $R$. solani.

In 1954, Townsend and Willetts divided the development of sclerotia into three stages: initiation, development, and maturation. The initiation stage is marked by the appearance of the 
55

56

57

58

59

60

61

62

sclerotium with white aerial mycelia entangled around the edges of the culture medium. In the development stage, the sclerotium becomes further entangled and increases in size along with secretion of a clear or tan-colored liquid on the surface. The maturation phase involves the accumulation of melanin in epidermal cells, and internal hardening. (Townsend and Willetts 1954). Substrates associated with these stages (initiation, development, and maturation) of a typical spherical sclerotium in filamentous fungi may show a differentiated structure, which can possibly insulate itself from environmental oxygen (Georgiou et al. 2006). One of the most unique features of the sclerotium is that it becomes inactive when the environmental conditions are not conducive for growth. In the mature stage of the sclerotium, it can survive for many years enduring extreme temperatures, desiccation, starvation, harmful irradiation, and biological degradation (Georgiou et al. 2006; Liang et al. 2010). Thus, understanding how the sclerotia form is important for crop disease control. However, the molecular mechanisms of sclerotia formation of $R$. solani have been poorly understood.

Therefore, to understand the molecular basis of sclerotia formation, we determined the transcriptomes of $R$. solani AG1-IA in the three important developmental stages (mycelium, sclerotia initiation, and sclerotia maturation) using RNA-sequencing (RNA-seq), which has been frequently applied to the discovery of the molecular mechanisms underlying pathogenic growth and development (Egan et al. 2012; Metzker 2010; O'Connell et al. 2012). Moreover, RNA-seq performs better DEG analysis compared to other high-throughput technologies (Wang et al. 2009). Transcriptome data were collected from the mycelium ( $4 \mathrm{~d}$ of growth), initial sclerotia (5 $\mathrm{d}$ of growth), and mature sclerotia (7 d of growth) of $R$. solani AG1-IA. Moreover, we examined the underlying pathways involved in sclerotonium formation. In particular, we tested the hypothesis that ROS are involved in the process.

ROS arises from mitochondrial oxidative metabolism and in the cellular response to xenobiotics, cytokines, and bacterial invasion. (Ray et al. 2012). The influence of ROS on molecular and biochemical processes as well as signal transduction pathways is well-established, which in turn affects cell proliferation and differentiation, leading to the death of fungi andother organisms (Allen \& Tresini 2000). ROS are more reactive than $\mathrm{O}_{2}$ in its ground state or triplet state $\left({ }^{3} \mathrm{O}_{2}\right)$. These ROS are dioxygen molecules in their excited singlet state forms $\left({ }^{1} \mathrm{O}_{2}\right)$ and partially reduced forms of oxygen, including superoxide radical ion and its protonated forms $\mathrm{HO}$. 2, hydroxyl radical (HO), and hydrogen peroxide $\left(\mathrm{H}_{2} \mathrm{O}_{2}\right)$ (Cui et al. 2011; Georgiou et al. 2006; Turrens 2003). Moreover, ROS play an important role in the regulation of cellular signaling pathways; for example, hyperoxidant states are the primary driving force for cell differentiation (Fang et al. 2002; Lara-Ortíz et al. 2003). Chet and Henis (1975) first proposed that the formation of sclerotia requires the participation of oxygen. However, addition of a hydroxyl radical scavenger to the culture medium of Sclerotinia sclerotiorum and R. solani was shown to suppress sclerotium formation (Georgiou et al. 2000). Therefore, to further understand this process, we measured the production of ROS during the three stages of sclerotium formation and determined the activities of key ROS- producing and ROS-scavenging enzymes during the process.

This work should help to identify the critical genes responsible for formation of the sclerotium 
96

97

98

99

100

101

102

103

104

105

106

107

108

109

110

111

112

113

114

115

116

117

118

119

120

121

122

123

124

125

126

127

128

129

of $R$. solani AG1-IA in maize toward understanding the underlying molecular mechanism. The generated results should provide a scientific foundation for the development of new strategies and targets toward the prevention of corn sheath blight.

\section{MATERIALS AND METHODS}

\section{Sample collection and preparation}

$R$. solani AG1-IA were collected from the Institute of Plant Immunology at Shenyang Agricultural University. This strongly pathogenic strain was screened as previously described (Tingting et al. 2013) and cultivated on potato dextrose agar (PDA), at a temperature of $26^{\circ} \mathrm{C}$. The $R$. solani AG1-IA were collected from PDA at different stages of maturation: mycelium, RWF9M (4 d of growth); sclerotial initiation, RWF9SI (5 d of growth); sclerotial maturation, RWF9S ( $7 \mathrm{~d}$ of growth) (Fig. 1). R. solani AG1-IA obtained were stored at $-80^{\circ} \mathrm{C}$. Three mycelia growth plates were used as three biological replicates for sclerotia formation and harvesting.

\section{RNA quantification}

RNA was extracted at the three developmental stages of the sclerotium (mycelium, sclerotium initiation, and sclerotium maturation) using TaKaRa MiniBEST Universal RNA Extraction Kit (TaKaRa, Shiga, Japan). RNA quality was assessed on $1 \%$ agarose gel. PrimeScript ${ }^{\mathrm{TM}}$ RT reagent kit with gDNA Eraser (Takara, Shiga, Japan) was used to synthesize the cDNA. Total RNA was treated with DNase to remove gDNA.

\section{Library preparation for transcriptome sequencing}

This study provided $3 \mu \mathrm{g}$ RNA per sample for on-machine sequencing, performed using a NEBNext ${ }^{\circledR}$ Ultra $^{\mathrm{TM}}$ RNA Library Prep Kit for Illumina ${ }^{\circledR}$ (New England Biolabs, USA). Using mRNA as a template, first-strand cDNA was synthesized using random hexamers, followed synthesize and purify second-strand cDNA. The purified second-strand cDNA was subjected to terminal repair. A tail was added, and the sequencing adapter was adaptor-ligated cDNA. Then, we produced the cDNA library by fragment size selection (150-200 bp).

\section{Clustering and sequencing}

In the cBot Cluster Generation, the samples were clustered using the TruSeq PE Cluster Kit v3cBot-HS (Illumina); after clustering, the library was sequenced to generate $125 \mathrm{bp} / 150 \mathrm{bp}$ pairedend reads on the Illumina HiSeq platform. All raw-sequence read were stored at Sequence Read Archive (SRP134130).

\section{Quality control}

The raw sequence obtained by sequencing contains low-quality reads with linkers. The lowquality reads and the reads with adapter or poly-N were removed from raw data. Then, we counted the quality-control indices (Q20, Q30) and GC content (Trapnell et al. 2010). 
130

131

132

133

134

135

136

137

138

139

140

141

142

143

144

145

146

147

148

149

150

151

152

153

154

155

156

157

158

159

160

161

162

163

\section{Read mapping to the reference genome}

We used TopHat v2.0.12 and aligned the clean reads with those from Rhizoctonia solani AG-3 Rhs1AP genome (Cubeta et al. 2014) (Trapnell et al. 2009).

\section{Quantification of gene expression levels}

HTSeq v0.6.1 software was used to analyze the gene expression level in each sample. The fragments per kilobase of transcript per million reads (FPKM) value of each gene was then calculated based on the length and depth (Trapnell et al. 2010).

\section{Differential expression analysis}

We used the DESeq R software package (1.18.0) to analysis DEGs of the three stages (3 replicates per stage) (Wang et al. 2009). The $\mathrm{P}$ values of differentially expressed genes were adjusted by Benjamin and Hochberg methods (P values < 0.05) (Anders \& Huber 2012; Benjamini \& Hochberg 1995).

\section{GO and KEGG analysis}

We used GO seq R package for correcting gene $(\mathrm{P}$-value $<0.05)$ and implemented GO enrichment analysis of DEGs (Young et al. 2010). We used KOBAS to analyse the KEGG of DEGs (Kanehisa et al. 2007; Mao et al. 2005).

\section{Determination of ROS production}

ROS generation in the mycelium, initial sclerotium, and mature sclerotium of $R$. solani AG1 IA was detected using dichloro-dihydrofluorescein diacetate ( $\mathrm{H}_{2}$ DCFDA) (Ezaki et al. 2000). Samples from the three stages were incubated with $100 \mu \mathrm{L}$ of $10 \mu \mathrm{M} \mathrm{H}_{2} \mathrm{DCFDA}$, for $90 \mathrm{~min}$. To indicate the extent of ROS production, the fluorescence staining intensity was evaluated by using a fluorescent enzyme labeling instrument SpectraMax Gemini®EM (Molecular Devices, USA) at an excitation wavelength of $488 \mathrm{~nm}$ and emission wavelengths of $530 \mathrm{~nm}$.

\section{Enzyme activity assays}

SOD enzyme activity was measured based on the method by Beauchamp et al. (1971) with minor modifications. To $100 \mathrm{mg}$ of fungi, $1 \mathrm{~mL}$ sodium phosphate buffer $(60 \mathrm{mM}, \mathrm{pH} 7.0)$ was added. The mixture was grinded to extract SOD. The activity of the SOD enzyme was determined by adding nitroblue tetrazolium (NBT) and measuring absorbance at $560 \mathrm{~nm}$ (Beauchamp et al. 1971). CAT enzyme activity was measured based on the method by Khanam et al. (2005) with minor modifications. To $100 \mathrm{mg}$ of fungi, $1 \mathrm{~mL}$ buffer $(16.6 \mathrm{mM}$ sodium phosphate buffer $(\mathrm{pH}$ 7.2), 0.6 mM EDTA, and $50 \mathrm{mg}$ PVP) was added and the mixture was grinded to extract CAT. The rate of consumption of hydrogen peroxide by the extract was assayed by measuring absorbance at $240 \mathrm{~nm}$. Next, cell membranes were separated from fungi by two-phase partitioning (Qiu et al. 2002). The superoxide radicals in the membrane were determined by 
treatment with $0.5 \mathrm{mM}$ XTT and $50 \mathrm{mM}$ NADPH followed by measurement of absorbance at $470 \mathrm{~nm}$. Thus, the total NOX enzyme activity was calculated (Able et al. 1998, Sutherland et al. 1997). All enzyme assays were performed in triplicates with two repetitions.

\section{Real-time PCR analysis}

Twelve specific primers and four reference gene-specific primers were obtained (Table S1). The amplification efficiency of primer pairs was determined as described by Radonić et al. (2004). Twelve DEGs were selected for RT-qPCR confirmation. The reaction mixture comprised $10 \mu \mathrm{L}$ SYBR (TaKaRa, Shiga, Japan), $2 \mu \mathrm{L}$ cDNA, $2 \mu \mathrm{L}$ primer pair, and $6 \mu \mathrm{L}$ water. The reaction was performed on a CFX-96 system (BioRad, USA). All samples were tested in triplicates with two repetitions. Mapping was performed by using SigmaPlot 12.5. We used geNorm, BestKeeper, NormFinder, and the deltaCt method to evaluate four candidate reference genes in three stages (Andersen et al. 2004, Vandesompele et al. 2002, Pfaffl et al. 2004, Silver et al. 2006) (Table S2).

\section{RESULTS}

\section{Transcriptome sequencing reads quality inspection and sample correlation analysis during sclerotia formation of $R$. solani AG1 IA}

Total RNAs were isolated from RWF9M (mycelium), RWF9SI (sclerotial initiation), and RWF9S (sclerotial maturation) (Fig. 1). From three biological replicates, sequences of nine samples were generated to achieve $477,763,884$ raw reads, ranging from 45.44 to 65.77 million reads per sample. After quality filtering, 462,318,502 clean reads remained, ranging between 44.15 and 63.84 million per sample. Based on the quality test of the clean reads (Table 1), the Q20 from all reads was between $95.87 \%$ and $97.12 \%$ while the Q30 was between $90.79 \%$ and $92.35 \%$. These results showed that the data quality of the transcriptome was high and suitable for transcriptome analysis. The use of triplicates was employed for each biological sample on the data of original reads for the correlation test.

Prior to the DEGs analysis, the pearson correlation coefficients between samples were determined by RNA-seq correlation analysis. As shown in Figure 2A, the Pearson correlation coefficient of each sample was over 0.90 , demonstrating good repeatability. The results of the RPKM distribution were showed in Fig. 2B and RPKM density distribution were showed in Fig. 2C.

\section{DEGs during sclerotia formation of $R$. solani AG1 IA}

To obtain the DEGs, the gene expression of sclerotial initiation and maturation were compared to the mycelial stage. In addition, the sclerotial maturation was compared to the sclerotial initiation stage. A total of 5016, 6433, and 5004 DEGs were identified in the sclerotial initiation vs. mycelial, sclerotial maturation vs. mycelial, and sclerotial maturation vs. sclerotial initiation comparisons, respectively (Fig. 3). A total of 276, 1229, and 614 DEGs were identified in the sclerotial initiation vs. mycelial, sclerotial maturation vs. mycelial, and sclerotial maturation vs. 
201

202

203

204

205

206

207

208

209

210

211

212

213

214

215

216

217

218

219

220

221

222

223

224

225

226

227

228

229

230

231

232

233

234

235

236

237

238

239

240

241

sclerotial initiation groups, respectively (Fig. 3A). Overall, 2381 and 2635 genes were upregulated and downregulated, respectively, between the sclerotial initiation and mycelial samples. In the volcano plot, a total of 2381, 3102, and 2505 DEGs were upregulated in sclerotial initiation vs. mycelial, sclerotial maturation vs. mycelial, and sclerotial maturation vs. sclerotial initiation comparisons, respectively. In contrast, a total of 2635, 3331, and 2499 DEGs were downregulated in sclerotial initiation vs. mycelial, sclerotial maturation vs. mycelial, and sclerotial maturation vs. sclerotial initiation comparisons, respectively.

Under the GO terms, the unigenes were found to be involved in molecular function, cellular component, and biological process. Interestingly, the number of unigenes associated with the ribosome was particularly high in all three comparisons (Fig. 4). For the GO terms of DEGs in the sclerotial initiation vs. mycelial group, the high number of unigenes associated with singleorganism metabolic, metabolic, and oxidation-reduction (Fig. 4). The sclerotial maturation vs. mycelial group were largely associated with biological process, metabolic process, oxidoreductase activity, and catalytic activity. In addition, the DEGs in the sclerotial maturation vs. sclerotial initiation group were largely associated with carbohydrate metabolic process, cellular protein metabolic process, phosphorus metabolic process, and structural molecule activity.

When the DEGs were searched against the KEGG pathway, 2185 (sclerotial initiation vs. mycelial), 2286 (sclerotial maturation vs. mycelial), and 1882 (sclerotial maturation vs. sclerotial initiation) DEGs with significant hits were returned. The top 20 pathways in three groups are listed (Table 2, Table 3, and Table 4). Cluster analysis (Fig. 5) revealed that the expression pattern for DEGs in sclerotial initiation and maturation differ from that of the mycelial stage, indicating a significant change in gene expression levels during sclerotia formation. Eight clusters were plotted with their expression patterns (Fig. 5A). Based on the obtained FPKM data for hierarchical cluster analysis (Fig. 5B). As shown in Figure 5, subcluster 1 included 2334 genes with down-regulated expression at both sclerotia stages, and the expression levels of these genes were slightly lower in the sclerotial initiation and maturation samples than those in the mycelial samples. The 1396 genes in subcluster 2 were up-regulated at the sclerotial initiation stage compared to the mycelial stage, and then down-regulated at the sclerotial stage in comparison to the sclerotial initiation stage. The 220 genes in subcluster 3 were down-regulated in both sclerotial stages, the expression levels in sclerotial maturation were lower than the other two stages. Subcluster 4 included 570 genes with up-regulated expression in both stages.

Subcluster 5 included 2511 genes with higher expressionin sclerotial maturation than in the other two stages. Subcluster 6 included 922 genes with higher expression in the mycelial stage than in the two sclerotial stages. Subcluster 7 included 586 genes with higher expression in the sclerotial initiation stage than the other two stages. The 26 genes in subcluster 8 were up-regulated at both stages, and the expression levels in sclerotial initiation were higher than those in the mycelial stage, whereas those of the sclerotial maturation stage were lower compared to the sclerotial initiation stage. The top 20 genes from each category were selected (Tables S3-S10), indicating that NADPH dehydrogenase, cytochrome P450, oxygen-dependent choline dehydrogenase, chitin synthase, O-methylsterigmatocystin oxidoreductase, CAT, and NADPH-P450 reductase 
242

243

244

245

246

247

248

249

250

251

252

253

254

255

256

257

258

259

260

261

262

263

264

265

266

267

268

269

270

271

272

273

274

275

276

277

278

279

play particularly important roles in sclerotial formation. The DEGs of each category were annotated in GO terms and searched against the KEGG pathway (Fig. S1, S2). The GO classification showed that a higher proportion of DEGs in subcluster 3, 4, and 8 are involved in oxidation-reduction process, oxidoreductase activity, and antioxidant activity. In subcluster 4, four genes ( $S O D, C A T$, putative protein disulfide-isomerase, and glutathione peroxidase) were classified in the antioxidant activity GO term, and $S O D$ and $C A T$ were predicted to be localized at the peroxisome.

\section{Oxidative stress responses}

To investigate the changes of ROS production during the formation of sclerotia, and changes of enzymes in ROS, the fluorescent dye $\mathrm{H}_{2}$ DCFDA was used to measure intracellular ROS levels in the three stages of $R$. solani AG1 IA development. As shown in Figure 6, ROS were detected in all three stages, although the intensity of fluorescence was much higher in the sclerotial initiation stage compared to the other two stages.

CAT, SOD, and NOX are the key to the production and removal of ROS in the formation of sclerotia. Therefore, we assayed these enzymes. NOX and SOD showed an initial increase in activity followed by a decrease. NOX enzymes participates in the production of superoxide, an important precursor of ROS, and SOD can convert superoxide radicals to hydrogen peroxide. Thus, the increase of NOX and SOD enzyme activities in the sclerotial initiation showed that the ROS level improved. By contrast, CAT activity decreased in all three stages, which indicates a reduction of ROS scavenging (Fig. 6). In addition, we treated the samples with the CAT inhibitor aminotriazole along with $\mathrm{H}_{2} \mathrm{O}_{2}$, and monitored sclerotial formation, which showed that $\mathrm{H}_{2} \mathrm{O}_{2}$ accumulation in the absence of CAT led to the early differentiation of sclerotia (Fig. S3, S4).

\section{Amplification specificity and efficiency}

The gel agarose results showed all primer bands at a single size range of 100-300 bp, and the melting curves had a single peak (Fig. S5-S9). The PCR efficiency (E) of all the primers was 94.9-109.5\%, and the correlation coefficients $\left(\mathrm{R}^{2}\right)$ were greater than 0.982 (Table $\mathrm{S} 1$ ). These results indicated that all primers could be used for subsequent RT-qPCR. Four reference genes were ranked by their average expression stability (M-value), as shown in Table S2.

Comprehensive analysis showed that 18sRNA was more stable compared with the RNA of actin, $\beta$-tubulin (TUB), and glyceraldehyde-3-phosphate dehydrogenase (GAPDH).

\section{Validation of gene expression level changes during sclerotia formation of $R$. solani AG1 IA} by RT-qPCR

The relative mRNA levels of 12 DEGs among the three stages were analyzed using RT-qPCR: 6phosphogluconate dehydrogenase (6PGD), galactinol synthase 7 (CLOS7), glucan 1,3-betaglucosidase, beta-galactosidase, NADH dehydrogenase, NADPH-P450 reductase, obtusifoliol 14-alpha demethylase (CYP51), SOD, NOX, oxygen-dependent choline dehydrogenase (bETA), chitin synthase $\mathrm{D}$, and phosphoadenosine phosphosulfate reductase (MET16). The expression level trends of most of these genes were consistent between the RNA-seq and RT-qPCR results 
280

281

282

283

284

285

286

287

288

289

290

291

292

293

294

295

296

297

298

299

300

301

302

303

304

305

306

307

308

309

310

311

312

313

314

315

316

317

318

319

(Fig. 7).

\section{DISCUSSION}

As far as we know, this is the first transcriptome analysis on maize sheath blight (R. solani AG1 IA) during the sclerotium formation process. Kwon et al. (2014) analyzed proteomic changes during the process of sclerotial formation of $R$. solani for 5 days, 7 days, and 10 days, respectively, and obtained 55 differentially expressed proteins, representing high expression of processing, cellular processes, amino acid metabolism, cell defense, and carbohydrate metabolism during the sclerotium formation. In line with these previous findings, through RNAseq analysis during the formation of the sclerotium, we identified 5000-6000 DEGs between the three stages from the mycelial stage to the sclerotial initiation and maturation stages, and the majority of the highly expression were involved in the oxidation-reduction process, carbohydrate metabolic process, catalytic activity, and oxidoreductase activity. The involvement of carbohydrate metabolism suggests thatcarbon sources are quickly consumed in sclerotial maturation. Therefore, the cell metabolism was reduced. In addition, six antioxidant enzymes (three SODs, dihydropteroate synthase, cytochrome C peroxidase, and alpha-ketoglutaratedependent taurinedioxygenase) of $R$. solani were differently expressed during sclerotial maturation (Kwon et al. 2014). In this study, SOD, dihydropteroate synthase, and cytochrome C peroxidase were found to be differentially expressed among all three stages, and more antioxidant genes were found to accumulate during the sclerotia formation process, including CAT, putative protein disulfide-isomerase, and glutathione peroxidase. These results showed that SOD and CAT may regulate ROS levels to maintain cellular redox homoeostasis within cells during sclerotial maturation.

Zheng et al. (2013) suggested that in traditional breeding strategies, no crops have high resistance to $R$. solani. Although some resistance traits have been found in rice and maize, no resistance genes have been identified and cloned. However, these authors fully sequenced the transcriptome of $R$. solani AG1 IA, providing a molecular basis for the breeding of rice plants with resistance to the disease (Zheng et al. 2013). Our present work thus largely expands the available tools for breeding disease-resistant maize through generation of a transcriptome library of the sclerotial maturation of $R$. solani AG1 IA.

Some genes have previously been linked to sclerotia formation. $R$. solani with knockout Gprotein $\alpha$ subunit showed a change in morphology, and the ability to form sclerotium was completely lost (Charoensopharat K et al. 2008). The osmotic stress response and microsclerotia formation of Verticillium dahliae were regulated by the mitogen-activated protein kinase gene (VdHog1) (Yonglin et al. 2016). We further identified a large number genes related to sclerotia formation using RNA-seq technology. Moreover, the cluster analysis revealed that the expression pattern of DEGs in the sclerotial initiation and maturation stages differed from that in the mycelial stage. Eight clusters were plotted with their expression patterns, revealing significant difference of previously identified sclerotial formation-related genes. In particular, subcluster 4 included 570 genes that were up-regulated in both sclerotia stages, which were highly enriched in GO terms related to peroxidase activity, oxidoreductase activity, and 
320

321

322

323

324

325

326

327

328

329

330

331

332

333

334

335

336

337

338

339

340

341

342

343

344

345

346

347

348

349

350

351

352

353

354

355

356

357

358

359

oxidation-reduction process, and KEGG pathways of peroxisome, glutathione metabolism, ascorbate, and aldarate metabolism. This suggests that antioxidants (e.g., glutathione, vitamin E, and ascorbic acid,) and enzymes (e.g., SOD, CAT) can change the balance of ROS and thus affect sclerotial formation.

Under hyperoxidant state, cells can be transformed between differentiated and undifferentiated states, and the ROS level is higher than that in antioxidation. In this state, the cells need to prevent premature entry into the hyperoxidant state. To prevent entry into this hyperoxidant state, cells via cell aggregation or fusion in the biogenesis of sclerotia, plasmodia, and sex organs (Georgiou et al. 2006). Moreover, we found that SOD and NOX activities were particularly high in the initiation stages, suggesting that the early developmental stage of sclerotium generates ROS in hyperoxidant state. In contrast, CAT activity decreased in the maturation stage, suggesting an increase in the consumption of oxygen molecules with a consequent blockage of oxygen molecules from entering the cells to balance the peroxide content. Thus, ROS is an important inducing factor for the growth of $R$. solani from the mycelium to sclerotium.

NOX1, SOD, CAT are considered to be involved in superoxide anion generation, fungal growth, and cell differentiation (Peraza \& Hansberg 2002; Georgiou et al. 2006). Kim et al. (2011) silenced NOX genes in S. sclerotiorum, $S s N O X 1$ and $S s N O X 2$, and found that the production of ROS among the mutant strains decreased, sclerotia could not be formed, and the synthesis of oxalic acid - an important pathogenic factor - also decreased significantly. Although the pathogenicity of the mutant was also significantly weakened, the gene silencing largely affected the formation of sclerotia rather than the pathogenicity of $S$. sclerotiorum. Furthermore, the mutation caused increased sensitivity to oxidative stress and significantly reduced the risk of disease (Veluchamy et al. 2012). Overall, our transcriptome analysis identified that changes in the expression of ROS-related genes, $N O X 1, S O D$, and $C A T$, are key events during sclerotia formation and development. These findings were confirmed with RT-qPCR validation for 12 genes, including MET16, 6PGD, and CLOS7 in addition to NOX1, SOD, and CAT, which may be involved in the production of superoxide anions, fungal growth, and cell differentiation. Thus, this study provides molecular-level evidence that active oxygen plays a role in $R$. solani AG1 IA sclerotia maturation, offering further insight into the molecular mechanism of sclerotial development.

\section{CONCLUSIONS}

In this study, we found a total of 8567 DEGs throughout $R$. solani AG1 IA sclerotial maturation, which were largely associated with oxidoreductase activity, carbohydrate metabolic process, and oxidation-reduction process. Cluster analysis revealed that genes showing the same trend in expression changes during sclerotia formation may have the same function. Twelve of these DEGs were confirmed using RT-qPCR, including $N O X 1$, SOD, and CAT, demonstrating a key role of oxidative stress through the production of superoxide anions, as well as general associations with fungal growth and cell differentiation. Based on our results, further studies should be performed to elucidate the specific metabolic pathways and functions during sclerotial development using modern techniques. The genes identified in this work only present the first 
360

361

362

363

364

365

366

367

368

369

370

371

372

373

374

375

376

377

378

379

380

381

382

383

384

385

386

387

388

389

390

391

392

393

394

395

396

397

398

step in revealing the underlying processes, but should provide a useful resource and targets for developing strategies toward the prevention and control of diseases caused by $R$. solani AG1 IA to improve crop productivity.

\section{Supplemental information}

Supplemental Tables all supplemental tables (Table S1-S10).

Supplemental Figures all supplemental figures (Figure S1-S10).

\section{REFERENCES}

Able AJ, Guest DI, Sutherland MW. 1998. Use of a new tetrazolium-based assay to study the production of superoxide radicals by tobacco cell cultures challenged with avirulent zoospores of Phytophthora parasitica var nicotianae. Plant Physiology. 117:491-499. DOI 10.1104/pp.117.2.491.

Allen R, and Tresini M. 2000. Oxidative stress and gene regulation. Free Radical Biology and Medicine 28:463-499. DOI 10.1016/S0891-5849(99)00242-7.

Anders S, and Huber W. 2012. Differential expression of RNA-Seq data at the gene level-the DESeq package. Heidelberg, Germany: European Molecular Biology Laboratory. Available at https://core.ac.uk/display/23260267 (accessed 30 September 2013)

Andersen CL, Jensen JL, Orntoft TF. 2004. Normalization of real-time quantitative reverse transcription-PCR data: a modelbased variance estimation approach to identify genes suited for normalization, applied to bladder and colon cancer data sets. Cancer Research 64:5245-5250. DOI 10.1158/0008-5472.CAN-04-0496.

Beauchamp C, Fridovich I. 1971. Superoxide dismutase: improved assays and an assay applicable to acrylamide gels. Analytical Biochemistry 44:276-287. DOI 10.1016/00032697(71)90370-8.

Benjamini Y, and Hochberg Y. 1995. Controlling the false discovery rate: a practical and powerful approach to multiple testing. Journal of the royal statistical society 57:289-300. DOI 10.2307/2346101.

Boland G, Melzer M, Hopkin A, Higgins V, and Nassuth A. 2004. Climate change and plant diseases in Ontario. Canadian Journal of Plant Pathology 26:335-350. DOI 10.1080/07060660409507151.

Charoensopharat K, Aukkanit N, Thanonkeo S, Saksirirat W, Thanonkeo P, Akiyama K. 2008. Targeted disruption of a $G$ protein $\alpha$ subunit gene results in reduced growth and pathogenicity in Rhizoctonia solani. World Journal of Microbiology \& Biotechnology 24:345-351. DOI 10.1007/s11274-007-9476-6.

Chet I, and Henis Y. 1975. Sclerotial morphogenesis in fungi. Annual review of phytopathology 13:169-192. DOI 10.1146/annurev.py.13.090175.001125.

Cubeta MA, Thomas E, Dean RA, Jabaji S, Neate SM, Tavantzis S, Toda T, Vilgalys R, Bharathan N, Fedorova-Abrams N, Pakala SB, Pakala SM, Zafar N, Joardar V, Losada L, Nierman WC. 2014. Draft genome sequence of the plant-pathogenic soil fungus Rhizoctonia solani anastomosis group 3 strain Rhs1AP. Genome Announcements 
399

400

401

402

403

404

405

406

407

408

409

410

411

412

413

414

415

416

417

418

419

420

421

422

423

424

425

426

427

428

429

430

431

432

433

434

435

436

437

438

439

5:e1072-14. DOI 10.1128/genomeA.01072-14.

Cui H, Kong Y, and Zhang H. 2011. Oxidative stress, mitochondrial dysfunction, and aging. Journal of signal transduction 2012: 646354. DOI 10.1155/2012/646354.

Egan AN, Schlueter J, and Spooner DM. 2012. Applications of next-generation sequencing in plant biology. Botanical Soc America 99: 175-185. DOI 10.3732/ajb.1200020.

Ezaki B, Gardner RC, Ezaki Y, Matsumoto H. 2000. Expression of aluminum-induced genes in transgenic arabidopsis plants can ameliorate aluminum stress and/or oxidative stress. Plant Physiology 122:657-665. DOI 10.1104/pp.122.3.657.

Fang G-C, Hanau R, and Vaillancourt L. 2002. The SOD2 gene, encoding a manganese-type superoxide dismutase, is up-regulated during conidiogenesis in the plant-pathogenic fungus Colletotrichum graminicola. Fungal Genetics and Biology 36:155-165. DOI 10.1016/S1087-1845(02)00008-7.

Georgiou CD, Patsoukis N, Papapostolou I, and Zervoudakis G. 2006. Sclerotial metamorphosis in filamentous fungi is induced by oxidative stress. Integrative and comparative Biology 46:691-712. DOI 10.1093/icb/icj034.

Georgiou CD, Tairis N, and Sotiropoulou A. 2000. Hydroxyl radical scavengers inhibit sclerotial differentiation and growth in Sclerotinia sclerotiorum and Rhizoctonia solani. Mycological Research 104:1191-1196. DOI 10.1017/S0953756200002707.

Kanehisa M, Araki M, Goto S, Hattori M, Hirakawa M, Itoh M, Katayama T, Kawashima S, Okuda S, and Tokimatsu T. 2007. KEGG for linking genomes to life and the environment. Nucleic acids research 36:D480-D484. DOI 10.1093/nar/gkm882.

Khanam NN, Ueno M, Kihara J, Honda Y, Arase S. 2005. Suppression of red light-induced resistance in broad beans to Botrytis cinerea by salicylic acid. Physiological \& Molecular Plant Pathology 66:20-29. DOI 10.1016/j.pmpp.2005.03.006.

Kim H-j, Chen C, Kabbage M, and Dickman MB. 2011. Identification and characterization of Sclerotinia sclerotiorum NADPH oxidases. Applied and environmental microbiology 77:7721-7729. DOI 10.1128/AEM.05472-11.

Kwon YS, Kim SG, Chung WS, Bae H, Jeong SW, Shin SC, Jeong M-J, Park S-C, Kwak Y-S, and Bae D-W. 2014. Proteomic analysis of Rhizoctonia solani AG-1 sclerotia maturation. Fungal biology 118:433-443. DOI 10.1016/j.funbio.2014.02.001.

Lara-Ortíz T, Riveros-Rosas H, and Aguirre J. 2003. Reactive oxygen species generated by microbial NADPH oxidase NoxA regulate sexual development in Aspergillus nidulans. Molecular microbiology 50:1241-1255. DOI 10.1046/j.1365-2958.2003.03800.x.

Liang Y, Rahman MH, Strelkov SE, and Kav NN. 2010. Developmentally induced changes in the sclerotial proteome of Sclerotinia sclerotiorum. Fungal biology 114:619-627. DOI 10.1016/j.funbio.2010.05.003.

Mao X, Cai T, Olyarchuk JG, and Wei L. 2005. Automated genome annotation and pathway identification using the KEGG Orthology (KO) as a controlled vocabulary. Bioinformatics 21:3787-3793. DOI 10.1093/bioinformatics/bti430.

Metzker ML. 2010. Sequencing technologies-the next generation. Nature reviews Genetics 11:31-46. DOI 10.1038/nrg2626. 
440

441

442

443

444

445

446

447

448

449

450

451

452

453

454

455

456

457

458

459

460

461

462

463

464

465

466

467

468

469

470

471

472

473

474

475

476

477

478

479

480

O'Connell RJ, Thon MR, Hacquard S, Amyotte SG, Kleemann J, Torres MF, Damm U, Buiate EA, Epstein L, and Alkan N. 2012. Lifestyle transitions in plant pathogenic Colletotrichum fungi deciphered by genome and transcriptome analyses. Nature genetics 44:1060-1065. DOI 10.1038/ng.2372.

Ogoshi A. 1987. Ecology and pathogenicity of anastomosis and intraspecific groups of Rhizoctonia solani Kuhn. Annual review of phytopathology 25:125-143. DOI 10.1146/annurev.py.25.090187.001013.

Peraza L, and Hansberg W. 2002. Neurospora crassa catalases, singlet oxygen and cell differentiation. Biological chemistry 383:569-575. DOI 10.1515/BC.2002.058.

Pfaffl MW, Tichopad A, Prgomet C, Neuvians TP. 2004. Determination of stable housekeeping genes, differentially regulated target genes and sample integrity: BestKeeper-excel-based tool using pair-wise correlations. Biotechnology Letters 26:509515. DOI 10.1023/B:BILE.0000019559.84305.47.

Priyatmojo A, Escopalao VE, Tangonan NG, Pascual CB, Suga H, Kageyama K, and Hyakumachi M. 2001. Characterization of a new subgroup of Rhizoctonia solani anastomosis group 1 (AG-1-ID), causal agent of a necrotic leaf spot on coffee. Phytopathology 91:1054-1061. DOI 10.1094/PHYTO.2001.91.11.1054.

Qiu QS, Guo Y, Dietrich MA, Schumaker KS, Zhu JK. 2002. Regulation of SOS1, a plasma membrane $\mathrm{Na}+/ \mathrm{H}+$ exchanger in Arabidopsis thaliana, by SOS2 and SOS3. PNAS 99:8436-8441. DOI 10.1073/pnas.122224699.

Radonić A. 2004. Guideline to reference gene selection for quantitative real-time PCR. Biochemical and Biophysical Research Communications 313:856-862 DOI 10.1016/j.bbrc.2003.11.177.

Ray PD, Huang B-W, and Tsuji Y. 2012. Reactive oxygen species (ROS) homeostasis and redox regulation in cellular signaling. Cellular signalling 24:981-990. DOI 10.1016/j.cellsig.2012.01.008.

Rush M, and Lee F. 1983. Rice sheath blight: a major rice disease. Plant Disease 67:829-832. DOI 10.1094/PD-67-829.

Silver N, Best S, Jiang J. Thein SL. 2006. Selection of housekeeping genes for gene expression studies in human reticulocytes using real-time PCR. BMC Molecular Biology 7:1-9. DOI 10.1186/1471-2199-7-33.

Sutherland MW, Learmonth BA. 1997. The tetrazolium dyes MTS and XTT provide new quantitative assays for superoxide and superoxide dismutase. Free Radical Research 27:283-289. DOI 10.3109/10715769709065766.

Tingting K, Xiaokun Z, Zenggui G, Shuo Z, Ming W, Xiaoxi W, Yanbo Z. 2013. Identification and Pathogenicity of mycelium Fusion Group of Corn sheath Blight in Northeast China. Journal of Maize Sciences 21:132-137. DOI 10.13597/j.cnki.maize.science.2013.04.002.

Townsend BB, Willetts HJ. 1954. The development of sclerotia of certain fungi. Transactions of the British Mycological Society 37:213-221. DOI org/10.1016/S0007-1536(54)800039 . 
481

482

483

484

485

486

487

488

489

490

491

492

493

494

495

496

497

498

499

500

501

502

503

504

505

506

507

508

509

510

511

512

513

Trapnell C, Pachter L, and Salzberg SL. 2009. TopHat: discovering splice junctions with RNA-Seq. Bioinformatics 25:1105-1111. DOI 10.1093/bioinformatics/btp120.

Trapnell C, Williams BA, Pertea G, Mortazavi A, Kwan G, Van Baren MJ, Salzberg SL, Wold BJ, and Pachter L. 2010. Transcript assembly and quantification by RNA-Seq reveals unannotated transcripts and isoform switching during cell differentiation. Nature biotechnology 28:511-515. DOI 10.1038/nbt.1621.

Turrens JF. 2003. Mitochondrial formation of reactive oxygen species. The Journal of physiology 552:335-344. DOI 10.1113/jphysiol.2003.049478.

Vandesompele J. 2002. Accurate normalization of real-time quantitative RT-PCR data by geometric averaging of multiple internal control genes. Genome Biology 3:research0034. DOI 10.1186/gb-2002-3-7-research0034.

Veluchamy S, Williams B, Kim K, and Dickman MB. 2012. The CuZn superoxide dismutase from Sclerotinia sclerotiorum is involved with oxidative stress tolerance, virulence, and oxalate production. Physiological and molecular plant pathology 78:14-23. DOI 10.1016/j.pmpp.2011.12.005.

Wang L, Feng Z, Wang X, Wang X, and Zhang X. 2009. DEGseq: an R package for identifying differentially expressed genes from RNA-seq data. Bioinformatics 26:136138. DOI 10.1093/bioinformatics/btp612.

Wang Z, Gerstein M, and Snyder M. 2009. RNA-Seq: a revolutionary tool for transcriptomics. Nature reviews genetics 10:57-63. DOI 10.1038/nrg2484.

Yonglin W, Longyan T, Dianguang X, Steven J, Shuxiao X, Chengming T. 2016. The mitogen-activated protein kinase gene, $\mathrm{VdHog} 1$, regulates osmotic stress response, microsclerotia formation and virulence in Verticillium dahliae. Fungal Genetics and Biology 88:13-23. DOI org/10.1016/j.fgb.2016.01.011.

Young MD, Wakefield MJ, Smyth GK, and Oshlack A. 2010. Gene ontology analysis for RNA-seq: accounting for selection bias. Genome biology 11:R14. DOI 10.1186/gb-201011-2-r14.

Zhao M, Zhang Z, Zhang S, Li W, Jeffers DP, Rong T, and Pan G. 2006. Quantitative trait loci for resistance to banded leaf and sheath blight in maize. Crop science 46:1039-1045. DOI 10.2135/cropsci2005.0166.

Zheng A, Lin R, Zhang D, Qin P, Xu L, Ai P, Ding L, Wang Y, Chen Y, and Liu Y. 2013. The evolution and pathogenic mechanisms of the rice sheath blight pathogen. Nature communications 4:1424. DOI 10.1038/ncomms2427. 
Figure 1

Three distinct stages in the formation of sclerotia of $R$. solani AGI IA.

(A) mycelium (4 d of growth); (B) initiation(5 $d$ of growth), and (C) maturation (7 d of growth). Photo credit: Bo Liu.

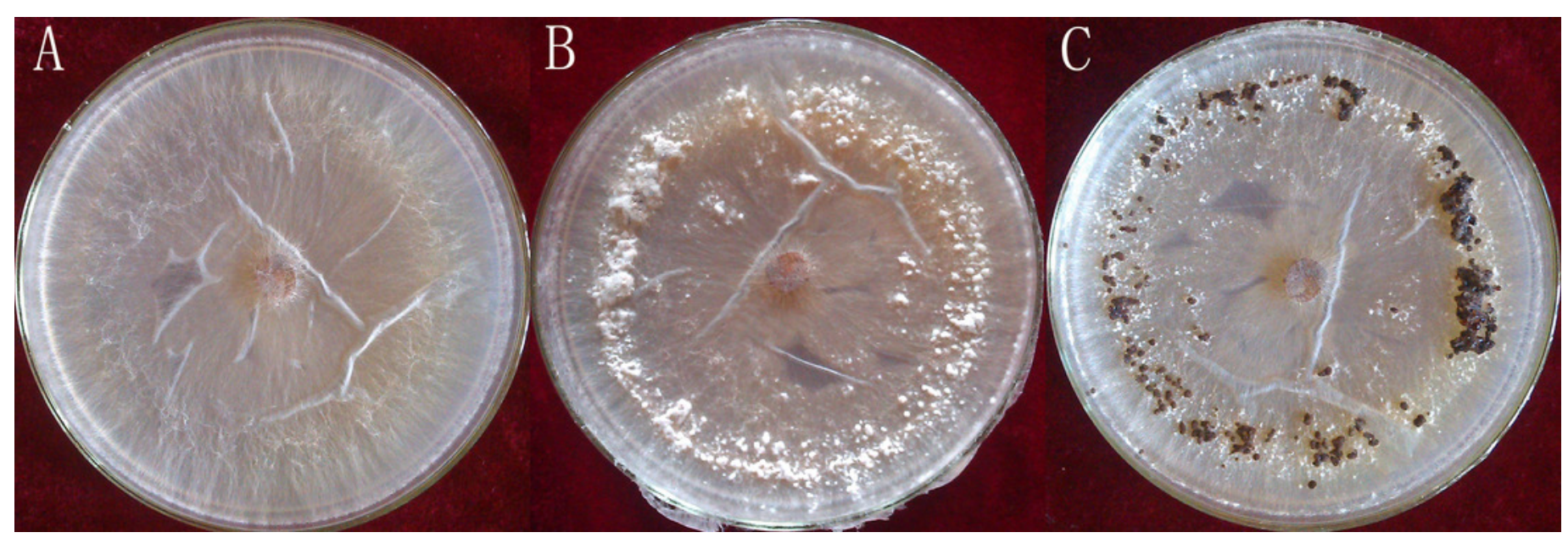


Figure 2

Bioinformatic analyses of RNA-seq data.
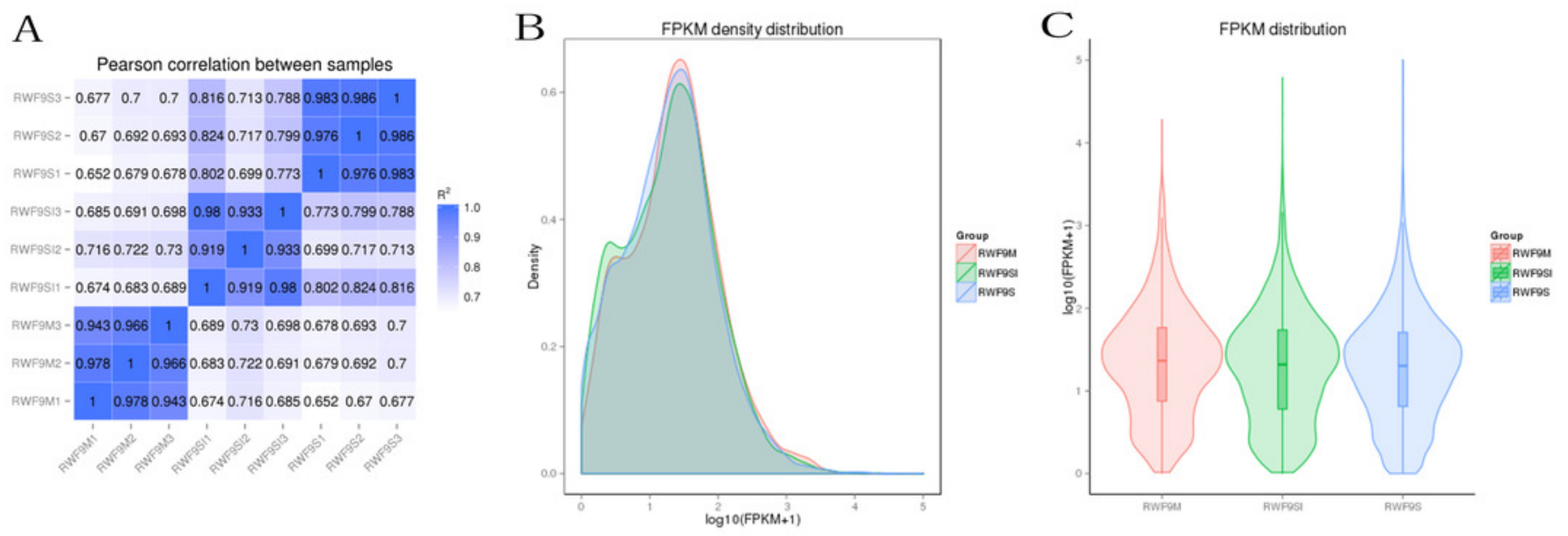
Figure 3

Differentially expressed genes analysis.

(A) Venn diagram of differentially expressed genes in the sclerotia formation of $R$. solani AG1 IA. (B-D) Volcano plot showing genes differently expressed between different libraries. The Qvalue for all plots was $<0.005$ and the absolute value of the $\log ^{2}$ Ratio $>1$ were used as the threshold to judge the significance of the difference in gene expression. Red points: genes up-regulate; Green points: genes down-regulated; Blue points: not DEGs.

A

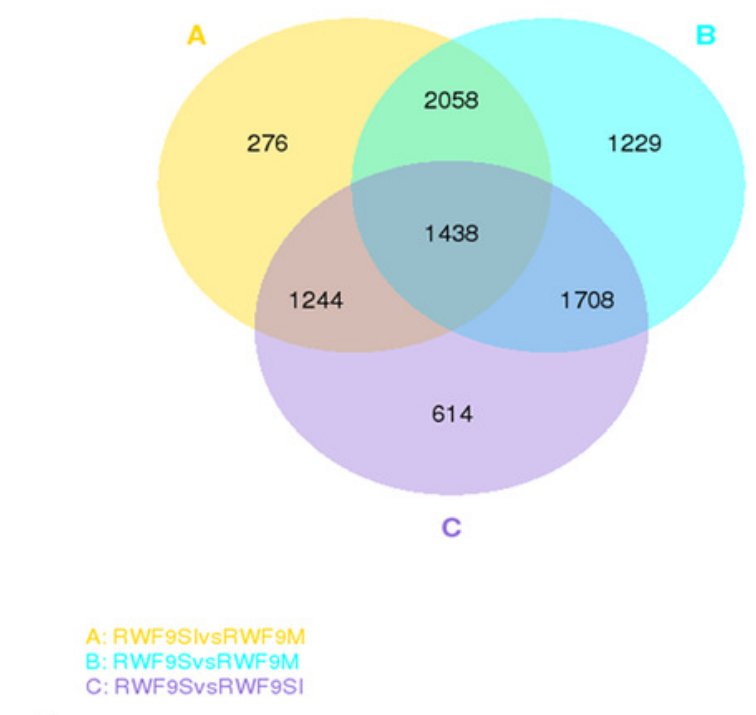

C

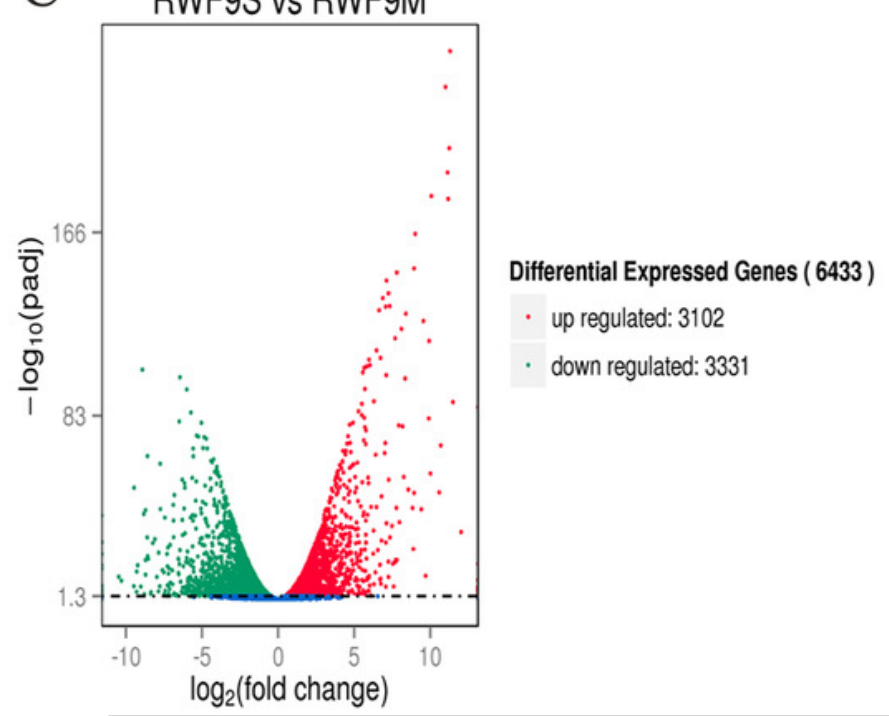

Peer] reviewing PDF | (2018:01:23251:2:0:NEW 26 May 2018)

$\mathrm{D}$
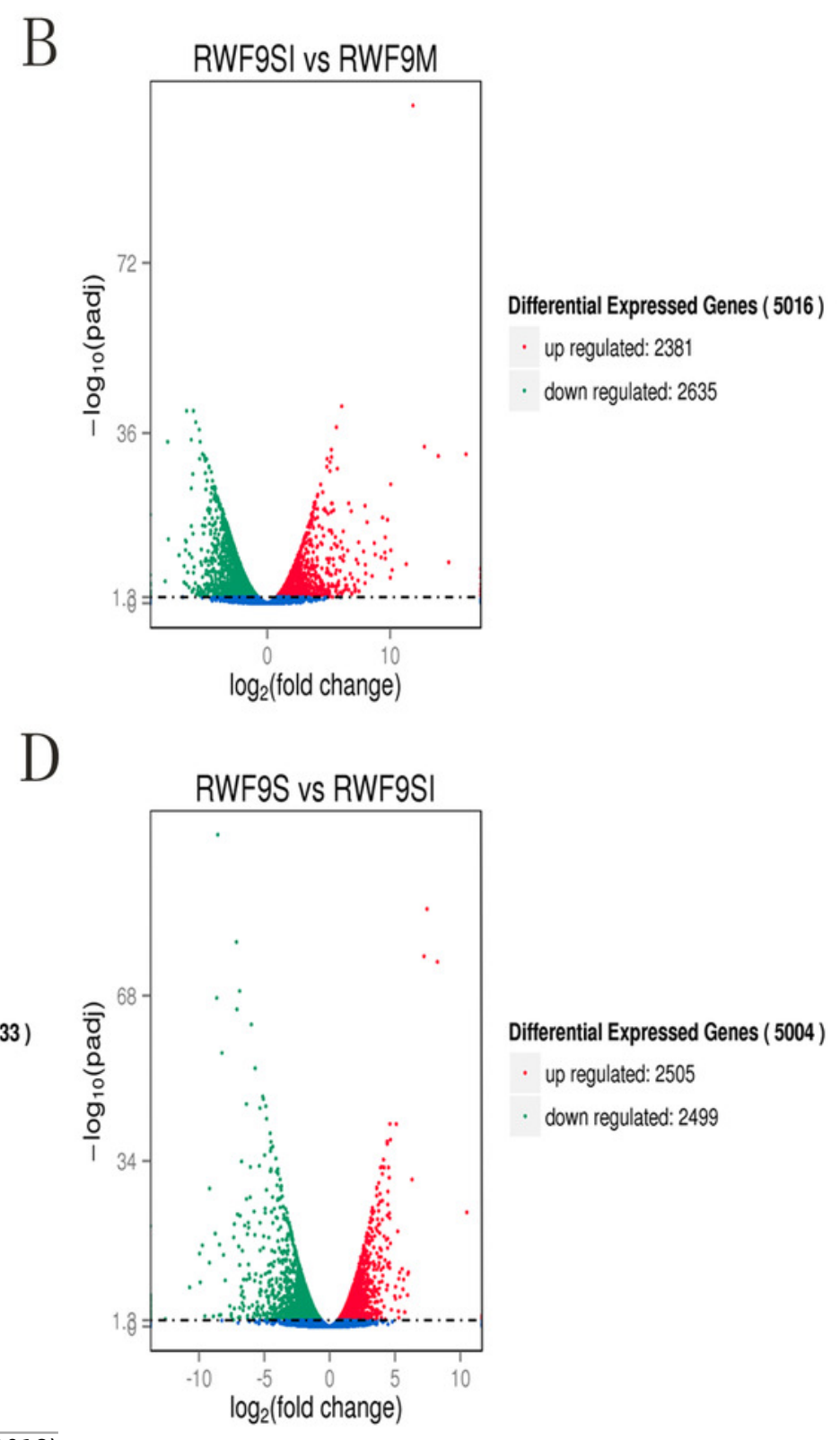

Differential Expressed Genes ( 5016)

up regulated: 2381

down regulated: 2635 


\section{Figure 4}

The difference of GO gene enrichment column, which reflects in the biological process, cellular component and molecular function.

The ordinate is the enrichment of GO term, the abscissa is the number of genes in the term. In different colors to distinguish between biological processes, cell component and molecular function.

A

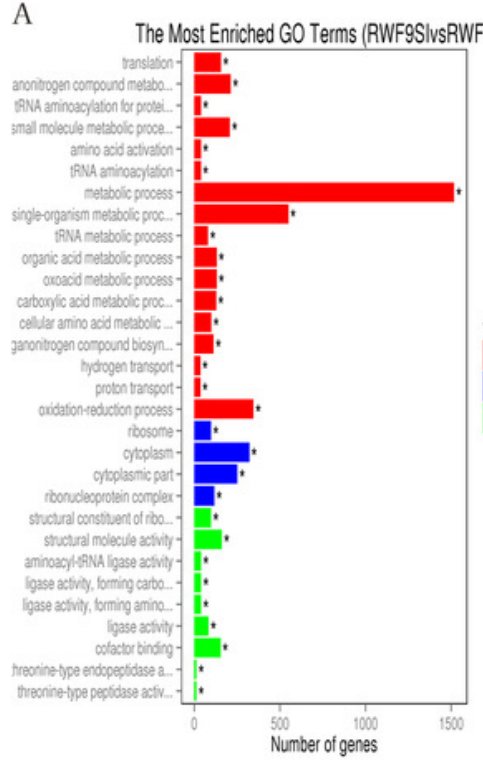

B

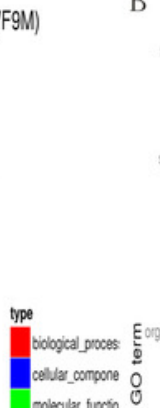$$
\text { sing ons }
$$

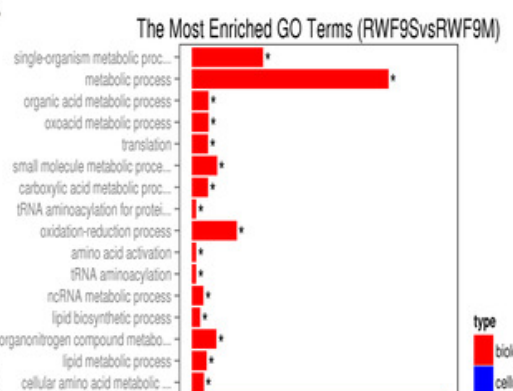

molecular functio

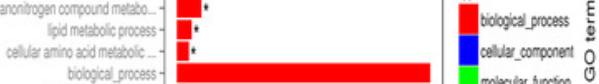

MinA metabolic procs

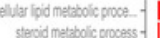

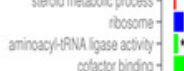

ligsse accivity, loming cato. . .

ligase activit toming antion. - .

coerzye binding -

cordred catse activy

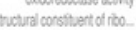

cateflic activy -

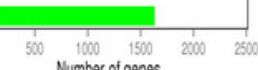

Number of genes
C

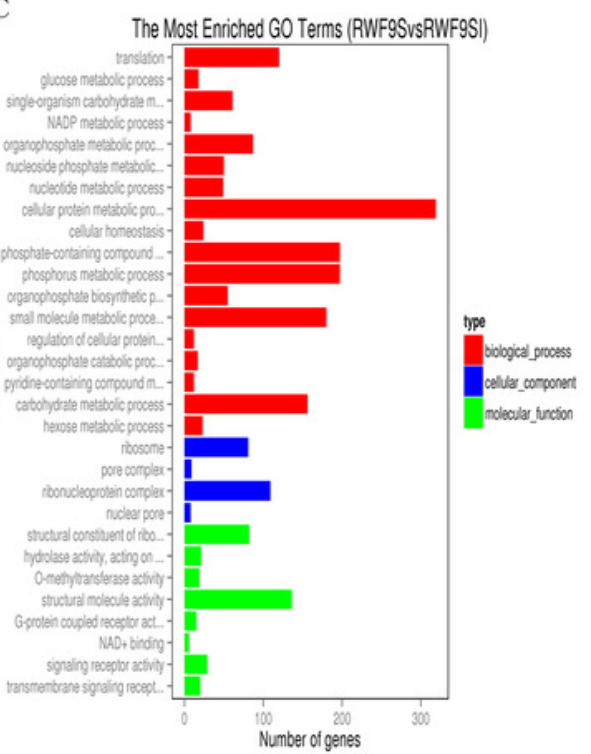




\section{Figure 5}

The clusters analysis.

(A) Expression patterns of the genes in the eight main clusters, namely subcluster 1- 8, corresponding to the heatmap.The gray is gene; The blue is gene tendency. (B) Hierarchical analysis and gene expression patterns of DEGs at three stages. Hierarchical cluster analysis of gene expression based on FPKM data. Low expression (blue) and high expression (red). The different stage is shown in the column and the transcriptional units in the rows. DEGs clustered in eight groups according to the similarity of their expression pattern. 
A subcluster_1, 2334 genes

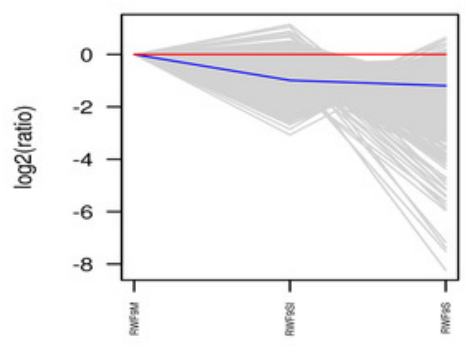

subcluster_3, 220 genes

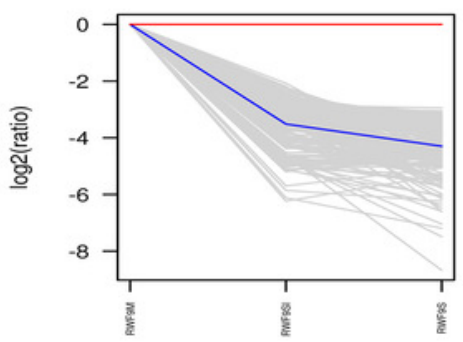

subcluster_5, 2511 genes

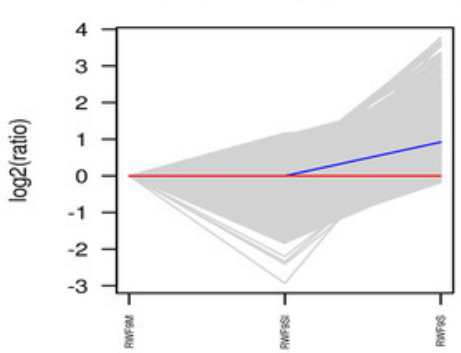

subcluster_7, 586 genes

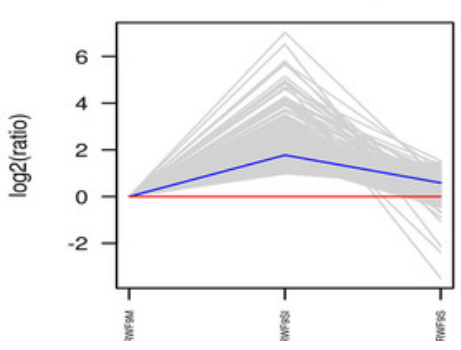

subcluster 2, 1398 genes

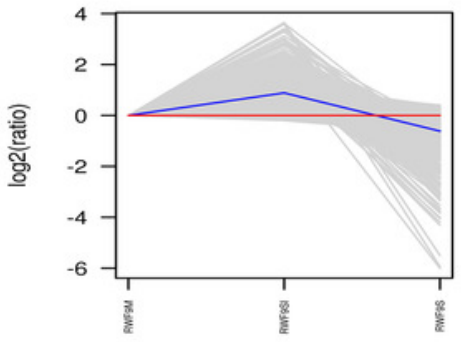

subcluster_4, 570 genes

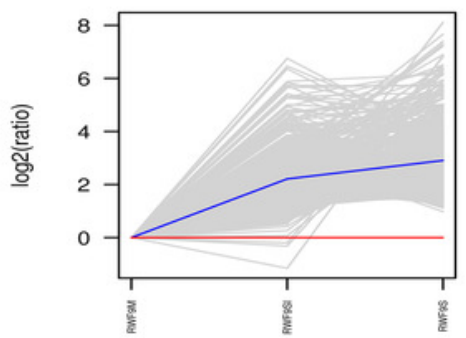

subcluster_6, 922 genes

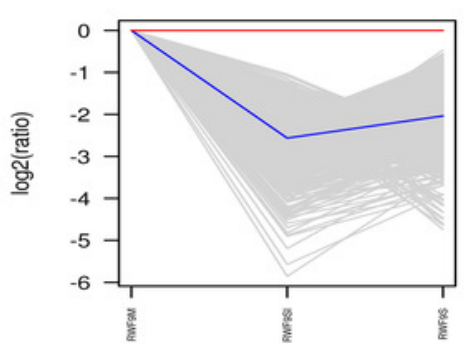

subcluster_8, 26 genes

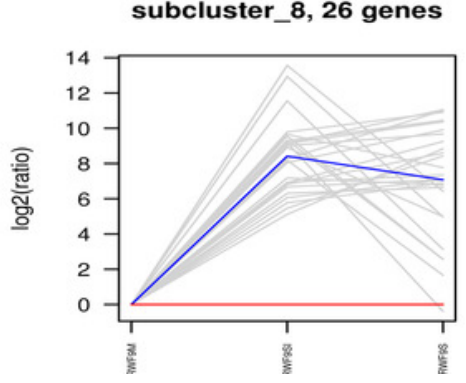

B Cluster analysis of differentially expressed genes

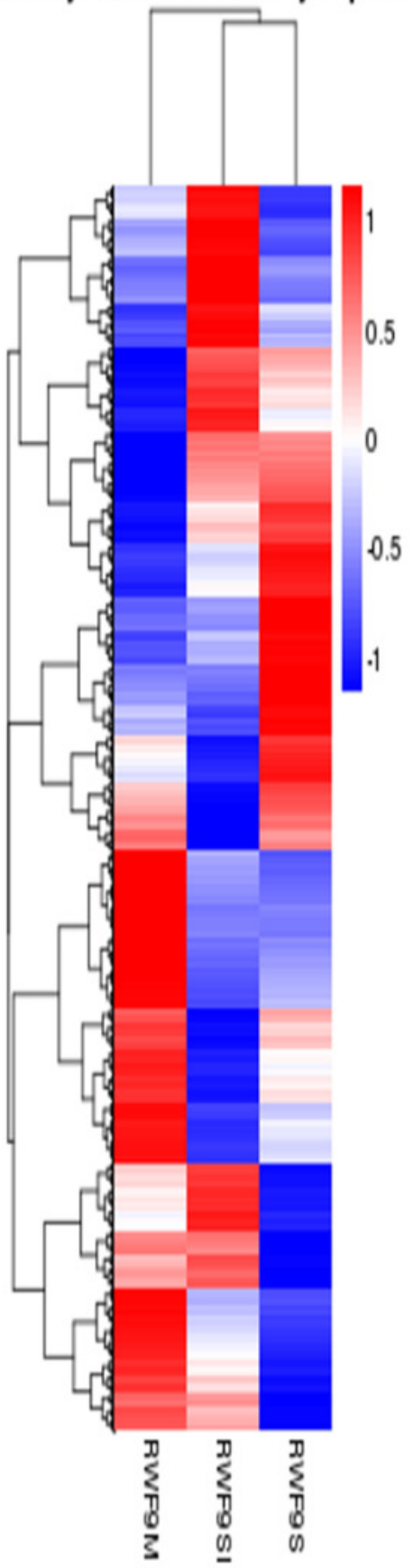


Figure 6

\section{Oxidative stress responses}

The ROS production levels and the enzyme activities of NOX1, SOD, and catalase (CAT) in three stages.M: mycelial SI: sclerotial initiation S: sclerotial maturation. $(p<0.01 * p<0.05, * *$ $p<0.01)$

A

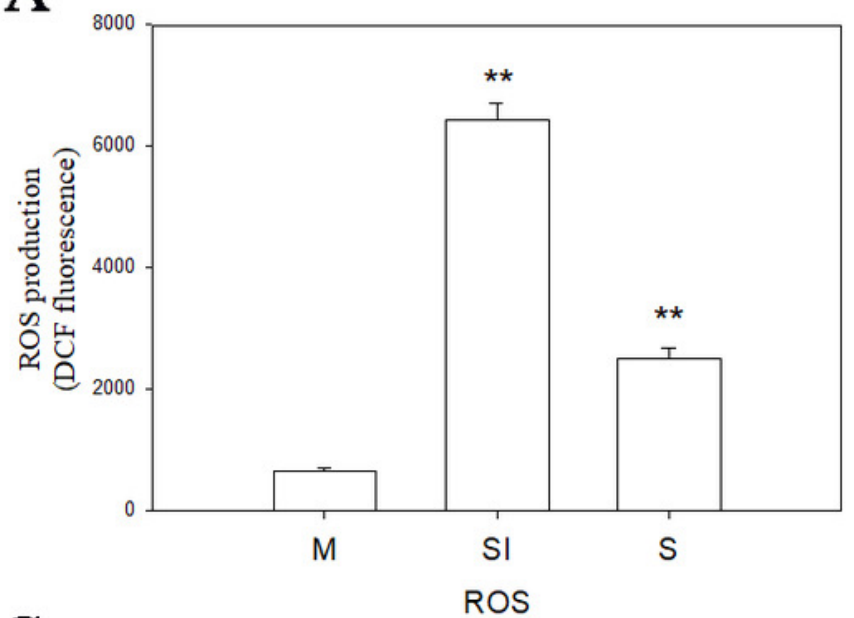

C

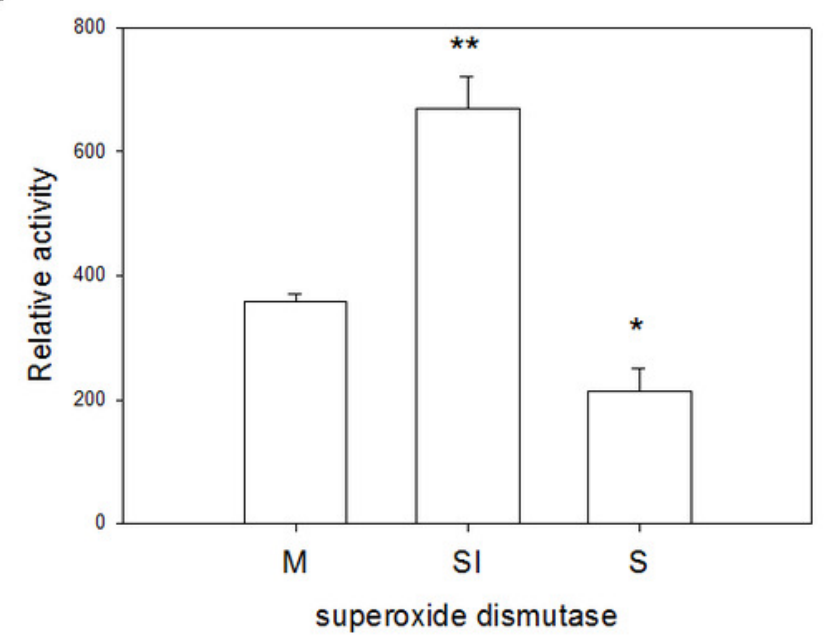

B

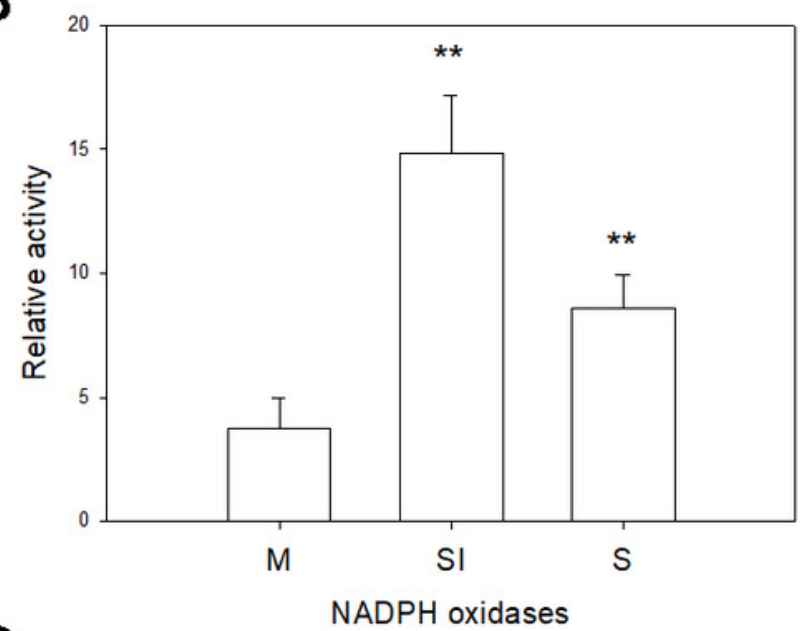

D

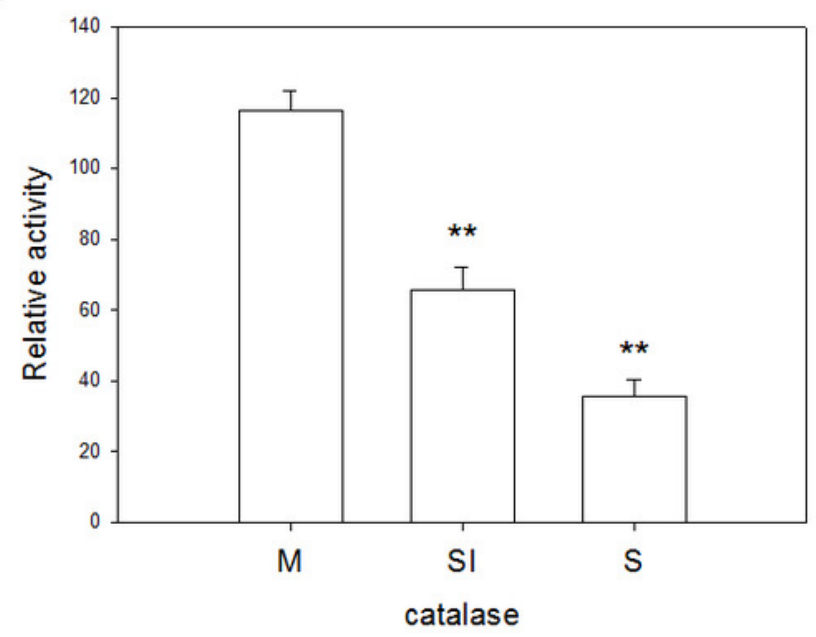


Figure 7

The expression level of twelve genes.

Real-time Quantitative PCR analysis of twelve genes in R. solani AG1 IA sclerotia maturation stage $\mathrm{S} 1, \mathrm{~S} 2$, S3. S1: RWF9M S2: RWF9SI S3: RWF9S.
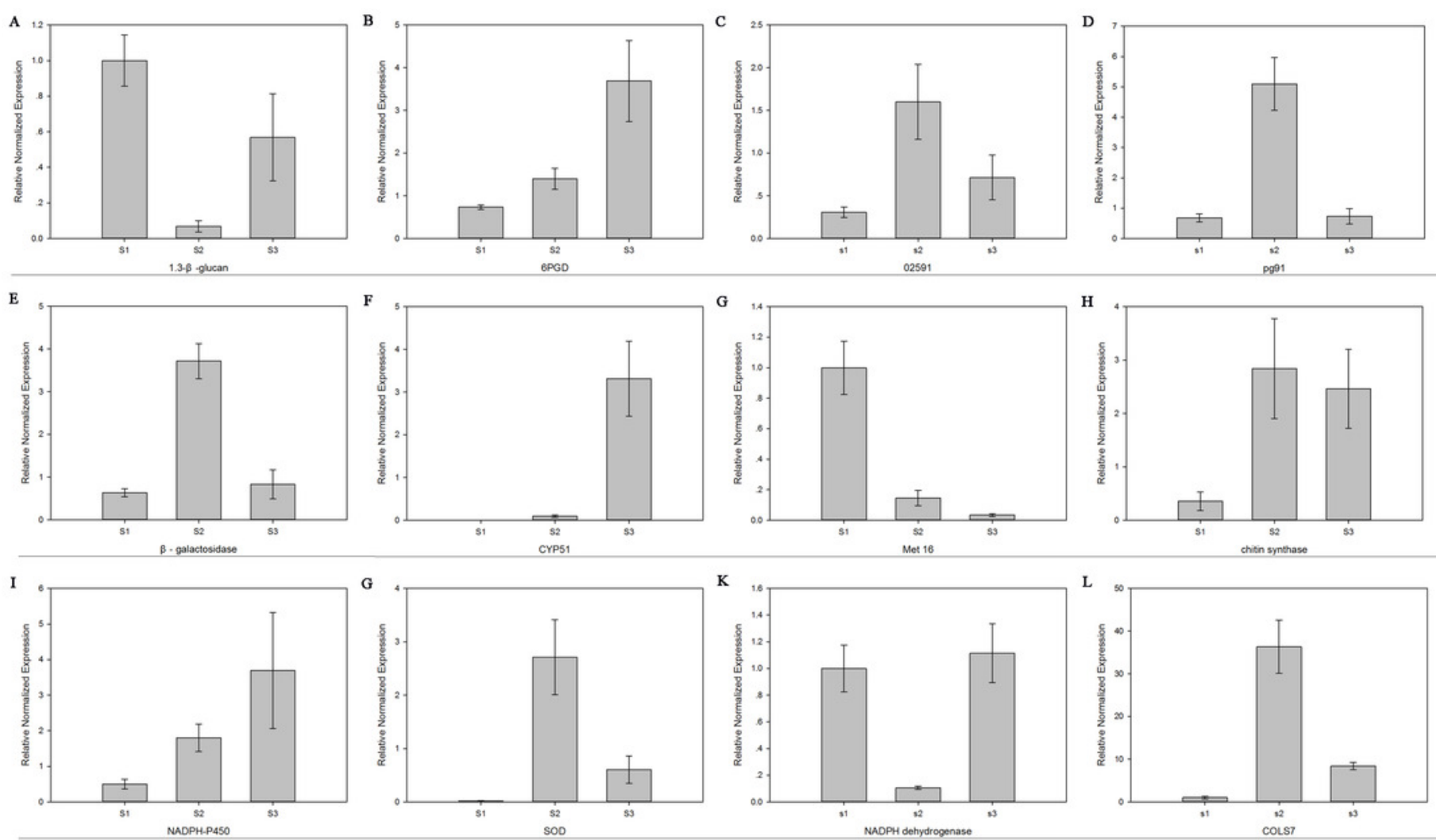


\section{Table $\mathbf{1}$ (on next page)}

Output statistics of sequencing.

1.RWF9M1 RWF9M2 RWF9M3 three biological replicates. 2.RWF9SI1 RWF9SI2 RWF9SI3 three biological replicates. 3.RWF9S1 RWF9S2 RWF9S3 three biological replicate. 


\begin{tabular}{cccccccc}
$\begin{array}{c}\text { Sample } \\
\text { name }\end{array}$ & Raw reads & $\begin{array}{c}\text { Clean } \\
\text { reads }\end{array}$ & $\begin{array}{c}\text { Clean } \\
\text { bases }\end{array}$ & $\begin{array}{c}\text { Error rate } \\
\mathbf{( \% )}\end{array}$ & $\begin{array}{c}\text { Q20 } \\
\mathbf{( \% )}\end{array}$ & $\begin{array}{c}\text { Q30(\% } \\
\text { ) }\end{array}$ & $\begin{array}{c}\text { GC content } \\
\text { (\%) }\end{array}$ \\
\hline RWF9M1 & 65770094 & 63841862 & $9.58 \mathrm{G}$ & 0.02 & 96.92 & 91.97 & 52.55 \\
RWF9M2 & 45449762 & 44158454 & $6.62 \mathrm{G}$ & 0.02 & 96.98 & 92.04 & 52.51 \\
RWF9M3 & 47018438 & 45744426 & $6.86 \mathrm{G}$ & 0.01 & 97.12 & 92.35 & 52.20 \\
RWF9SI1 & 52198062 & 49959440 & $7.49 \mathrm{G}$ & 0.02 & 95.92 & 90.83 & 51.73 \\
RWF9SI2 & 50834824 & 48902210 & $7.34 \mathrm{G}$ & 0.02 & 96.33 & 91.35 & 52.27 \\
RWF9SI3 & 49758464 & 47654054 & $7.15 \mathrm{G}$ & 0.02 & 95.87 & 90.79 & 51.71 \\
RWF9S1 & 54806008 & 53216446 & $7.98 \mathrm{G}$ & 0.02 & 96.92 & 91.94 & 51.10 \\
RWF9S2 & 52379712 & 50897498 & $7.63 \mathrm{G}$ & 0.02 & 96.99 & 92.08 & 51.09 \\
RWF9S3 & 59548520 & 57944112 & $8.69 \mathrm{G}$ & 0.01 & 97.11 & 92.33 & 51.23 \\
\hline
\end{tabular}


Table 2 (on next page)

Kegg RWF9SI vs. RWF9M. 
Kegg pathway

Metabolic pathways

Biosynthesis of secondary metabolites

Biosynthesis of amino acids

Ribosome

Carbon metabolism

RNA transport

Protein processing in endoplasmic reticulum

Oxidative phosphorylation

Spliceosome

Glycolysis / Gluconeogenesis

Cysteine and methionine metabolism

Arginine and proline metabolism

Pyruvate metabolism

2-Oxocarboxylic acid metabolism

Proteasome

Cell cycle - yeast

mRNA surveillance pathway

Purine metabolism

Glycine, serine and threonine metabolism

Valine, leucine and isoleucine degradation

Number of Unigenes

Percentage

\begin{tabular}{lc}
454 & 20.77 \\
195 & 8.92 \\
99 & 4.53 \\
91 & 4.16 \\
84 & 3.84 \\
49 & 2.24 \\
44 & 2.01 \\
43 & 1.97 \\
38 & 1.74 \\
36 & 1.65 \\
35 & 1.6 \\
34 & 1.56 \\
34 & 1.56 \\
32 & 1.46 \\
31 & 1.42 \\
31 & 1.42 \\
30 & 1.37 \\
30 & 1.37 \\
28 & 1.28 \\
26 & 1.19 \\
\hline
\end{tabular}




\section{Table $\mathbf{3}$ (on next page)}

Kegg RWF9S vs. RWF9M. 
Kegg pathway

Metabolic pathways

Biosynthesis of secondary metabolites

Biosynthesis of amino acids

Ribosome

Carbon metabolism

RNA transport

Protein processing in endoplasmic reticulum

Glycolysis / Gluconeogenesis

Cell cycle - yeast

Oxidative phosphorylation

Pyruvate metabolism

Arginine and proline metabolism

Purine metabolism

Spliceosome

Cysteine and methionine metabolism

Glycine, serine and threonine metabolism

Proteasome

Ribosome biogenesis in eukaryotes

Glutathione metabolism

Valine, leucine and isoleucine degradation
Number of Unigenes

498

219

97

85

82

51

44

42

39

38

37

36

34

31

30

29

29

29

28

28
Percentage

21.78

9.58

4.24

3.72

3.59

2.23

1.92

1.84

1.71

1.66

1.62

1.57

1.49

1.36

1.31

1.27

1.27

1.27

1.22

1.22 


\section{Table 4(on next page)}

Kegg RWF9S vs. RWF9SI. 
Kegg pathway

Metabolic pathways

Biosynthesis of secondary metabolites

Ribosome

Biosynthesis of amino acids

Carbon metabolism

RNA transport

Protein processing in endoplasmic reticulum

Spliceosome

Starch and sucrose metabolism

Cell cycle - yeast

Purine metabolism

Pyrimidine metabolism

Glycolysis / Gluconeogenesis

Meiosis - yeast

mRNA surveillance pathway

Oxidative phosphorylation

Arginine and proline metabolism

Peroxisome

N-Glycan biosynthesis

Amino sugar and nucleotide sugar metabolism
Number of Unigenes

342

134

80

55

49

47

45

45

37

37

37

28

27

27

26

26

25

25

23

23
Percentage

18.17

7.12

4.25

2.92

2.6

2.5

2.39

2.39

1.97

1.97

1.97

1.49

1.43

1.43

1.38

1.38

1.33

1.33

1.22

1.22 